recombinase (FVB/N genetic background $)^{11}$ and microinjection of a circularized Cre recombinase-expressing plasmid into $R f n g^{\Delta l-8 n e o}$ heterozygote fertilized eggs (129P2/OlaHsd $\times$ C57BL/6J hybrid background). In contrast to Rfng ${ }^{\Delta 1-8 n e o}$ homozygotes, $R f n g^{\Delta l-8}$ homozygotes on both genetic backgrounds were viable and were morphologically wild type (total $n=172$; Fig. 1f, i, l). We conclude that $R f n g$ function is not essential for AER formation or for proximal-distal limb outgrowth ${ }^{12}$. The absence of a limb phenotype in the $R f n g$ null allele may be due to functional overlap between Fringe family members.

We suggest that the phenotypes associated with the introduction of a PGKneoselection cassette into the $R f n g$ locus ( $R f n g^{\Delta l-8 n e o}$ allele) are caused by interference with the proper expression of a neighbouring gene or genes whose expression overlaps with that of $R f n g$ and are required for neural tube, craniofacial and limb development. There have been previous examples in which PGKneo effects have been examined. In such cases, the target genes have been part of a related gene complex that shares both regulatory control and functions (for example, the myogenic regulatory factors, HoxD and globin gene clusters ${ }^{13}$ ).

Our findings with $R f n g$-targeted alleles may be relevant for interpreting results from two $L f n g$-targeted alleles, $L f n g^{l a c Z}$ and $L f n g^{\text {Aneo }}$ (refs 9,10). In both of these Lfng gene-targeted alleles, the PGKneo-selection cassette was not excised from the locus ${ }^{9,10}$. The Lfng-targeted mutants exhibited phenotypic defects restricted to somitogenesis; however, there were unexplained differences in somite epithelialization. This phenotypic difference was attributed to either differences in genetic background or to differences in the design of the targeting constructs, although both targeted alleles were thought to be null alleles ${ }^{9,10}$.

With these Lfng alleles, it might therefore be possible that the PGKneo cassette affects the expression of a neighbouring gene(s) (perhaps paralogous between fringe family members). One such candidate is Uncx4.1, a paired type homeodomain gene that is physically linked to $L f n g$ and whose expression in the caudal half of condensing somites is altered in the Lfng mutants ${ }^{9,10,14}$. Our findings reinforce the importance of understanding the increasingly apparent presence of embedded and shared regulatory elements in genomes ${ }^{11}$.

Jennifer L. Moran, John M. Levorse,

Thomas F. Vogt

Department of Molecular Biology,

Lewis Thomas Laboratory, Princeton University,

Princeton, New Jersey 08544, USA

e-mail: tvogt@molbio.princeton.edu

1. Shubin, N., Tabin, C. \& Carroll, S. Nature 388, 639-648 (1997).

2. Irvine, K. D. \& Vogt, T. F. Curr. Opin. Cell Biol. 9, 867-876 (1997).

NATURE | VOL 399 | 24 JUNE 1999 | www.nature.com
3. Panin, V. M., Papayannopoulus, V., Wilson, R. \& Irvine, K. D. Nature 387, 908-912 (1997).

4. Laufer, E. et al. Nature 386, 366-373 (1997).

5. Rodriguez-Esteban, C. et al. Nature 386, 360-366 (1997)

6. Wu, J. Y., Wen, L., Zhang, W.-J. \& Rao, Y. Science 273, 355-358 (1996).

7. Johnston, S. H. et al. Development 124, 2245-2254 (1997).

8. Cohen, B. et al. Nature Genet. 16, 283-288 (1997).

9. Zhang, N. \& Gridley, T. Nature 394, 374-377 (1998).

10. Evrard, Y. A. et al. Nature 394, 377-381 (1998)

11. Lakso, M. et al. Proc. Natl Acad. Sci. USA 93, 5860-5865 (1996). 12. Christen, B. \& Slack, J. M. W. Nature 395, 230-231 (1998).

13. Olson, E. N., Arnold, H. H., Rigby, P. W. J. \& Wold, B. J. Cell 85, 1-4 (1996).

14. Moran, J. L. et al. Mamm. Genome 10, 535-541 (1999).

Zhang and Gridley reply - We too have created mice that have a large deletion in the Radical fringe gene and investigated the resulting effect on their development. Our results agree with those of Moran et al. in showing that $R f n g$ is not an essential gene in mice, but they do not support the idea that the presence of a neo-selection cassette inserted into the $R f n g$ locus invariably leads to embryonic lethality.

We constructed and analysed a targeted mutation of the Rfng gene by using a targeting vector in which a section of the $R f n g$ genomic sequence, encoding the region from amino acid 52 of the Rfng protein through to the end of the Rfng gene, was deleted. The vector contained a neo-selection cassette in the same transcriptional orientation as the Rfng gene. Mice homozygous for our Rfng mutant allele formed viable, morphologically normal adults at the expected mendelian frequencies (34 of 126 homozygotes $(27 \%)$ on a mixed C57BL/6J $\times 129 /$ SvImJ background, and 3 of 16 homozygotes (19\%) on an inbred 129/SvImJ background). The absence of wild-type $R f n g$ transcripts in our $R f n g$ homozygous-mutant adult mice was confirmed by northern blotting.

It is possible that a difference in the construction of the two targeted Rfng alleles could explain the different mouse phenotypes generated by Moran et al. - the orientations of the neo-selection cassette were not the same in the two targeted Rfng alleles, for instance. Reversing the orientation of the neo sequence for transcription creates a potent splice-acceptor site . $^{1,2}$.

In considering the implications of their finding for the interpretation of Lfng mutant phenotypes ${ }^{3,4}$, Moran et al. correctly point out that, in some instances, the presence of a neo-selection cassette in a targeted allele can affect the transcription of other genes, and hence the mutant phenotypes they generate. To confirm that the mutant phenotype is independent of the presence of a neo cassette in the two Lfng mutant alleles, a targeted $L f n g$ allele constructed without a neo cassette needs to be tested.

However, we believe that it is unlikely that the neo cassette in the two Lfng mutant alleles is responsible for the alterations in Uncx4.1 transcription or for the defects in somite formation that we detected in $L f n g$ homozygous-mutant embryos. The spontaneous mouse mutant pudgy $(p u)$ is caused by a frameshift mutation in the Dll3 gene ${ }^{5}$, which encodes a ligand for the Notch family of proteins. This frameshift leads to premature truncation of the Dll3 protein ${ }^{5}$. As homozygous Dll $3^{p u} / D l l 3^{p u}$ mutant embryos show defects in somite formation very like those seen in Lfng mutant embryos, we compared Uncx4.1 transcription in both $D l l 3^{p u} / D l l 3^{p u}$ and $L f n g^{\text {lacZ }} /$ ffng $^{\text {lacZ }}$ mutant embryos and found that the Uncx4.1 expression pattern was similarly disorganized in both mutants. Given that the presence of a neo cassette does not alter Uncx4.1 expression in $D l l 3^{p u} / D l l 3^{p u}$ embryos, the simplest explanation is that the alterations in Uncx4.1 expression and somite formation in $L f n g^{\text {lacZ }} / L f n g^{\text {lacz }}$ mutant embryos are caused by perturbations of the Notch signalling pathway, and not by the presence of a neo cassette in the $L f g^{\mathrm{lacZ}}$ mutant allele.

Nian Zhang, Thomas Gridley

The Jackson Laboratory,

Bar Harbor, Maine 04609-1500, USA

e-mail: gridley@jax.org

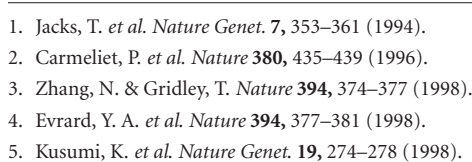

\section{Risk of collisions for constellation satellites}

The increasing quantity of debris orbiting the Earth is causing concern for space agencies. At the average collision velocity of 10 $\mathrm{km} \mathrm{s}^{-1}$, even projectiles $1 \mathrm{~cm}$ across can damage satellites. Impacts have a significant chance of occurring, especially for large structures that remain in orbit for a long time $^{1}$. Particularly at risk are satellites at altitudes of about 800 and 1,400 km, where there is already a high density of orbiting bodies and atmospheric drag is not effective in removing small fragments. We find that the dynamical architecture of satellite constellations, several of which are to be launched over the next decade, makes them particularly vulnerable to the consequences of an impact, which may set up a kind of chain reaction, triggering more collisions.

There are currently about $10^{4}$ objects larger than $10-20 \mathrm{~cm}$ across orbiting the Earth, with $10^{5}$ objects more than $1 \mathrm{~cm}$ across, and $10^{7}$ bodies exceeding $1 \mathrm{~mm}$ in diameter. These have a total mass of about $3 \times 10^{6} \mathrm{~kg}$ and a cross-section of $4 \times 10^{4} \mathrm{~m}^{2}$ (refs 2,3), and the average flux (the expected impact rate per projectile and per unit target cross-section) is about $3 \times 10^{-10} \mathrm{~m}^{-2} \mathrm{yr}^{-1}$. In the long term, debris from collisions with 


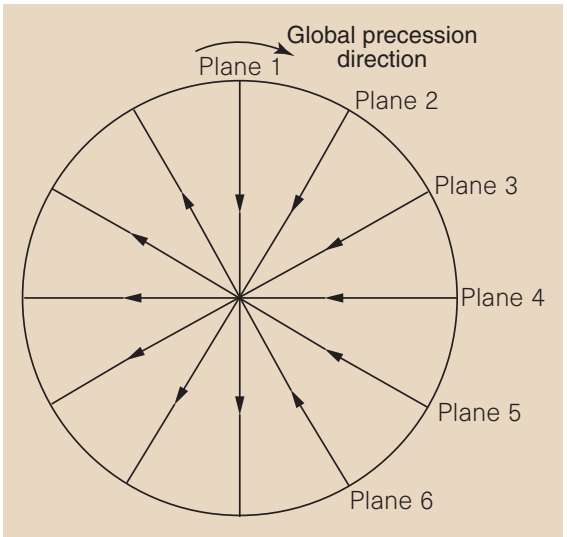

Figure 1 The orientation of the six orbital planes of an Iridium-like constellation, as seen from the celestial north pole. The revolution of the satellites is indicated by the arrows. Note the 'counter-rotating' planes 1 and 6 . If a satellite orbiting in either of these planes is disrupted, its fragments will pose an impact hazard much higher than average to the satellites in the other plane.

these objects may irreversibly pollute portions of near-Earth space ${ }^{4,5}$.

Satellite constellations, consisting of tens to hundreds of satellites orbiting at about the same altitude, will be launched into orbit over the next ten years, mainly for global telecommunication purposes ${ }^{6}$. One of these, the Iridium system, is already operational. It consists of 66 satellites (plus six spares) orbiting in six different orbital planes at an altitude of about $780 \mathrm{~km}$ and with an inclination of 86.4 degrees. The wet mass of each satellite is $667 \mathrm{~kg}$. Although some measures have been taken to minimize the risk of impact, the possibility of disruptive collisions cannot be ruled out, as the selected altitude corresponds to a peak of the expected debris flux. Assuming a catastrophic threshold for the impact specific energy of $4.5 \times 10^{3} \mathrm{~J} \mathrm{~kg}^{-1}$, as inferred from laboratory experiments on spacecraft mock-ups ${ }^{7}$, the flux of potentially shattering fragments at $800 \mathrm{~km}$ is about $10^{-5} \mathrm{~m}^{-2}$ $\mathrm{yr}^{-1}$. For a constellation cross-section of about $10^{3} \mathrm{~m}^{2}$, the risk of a catastrophic impact is about $10 \%$ per decade.

The architecture of such a constellation will make a break-up event particularly dangerous, owing to the spreading of the resulting fragment swarms, on a timescale intermediate between those analysed in previous studies (several hours ${ }^{8}$ and several decades $^{9}$ ). This is particularly true when differential precession of the orbits leads the fragments to encounter satellites revolving around the Earth in the opposite direction (Fig. 1); this makes head-on collisions possible, with higher impact speeds and greater collision probability.

Results of a simulation of an Iridium satellite being broken up by a 1-kg projectile at a relative speed of $10 \mathrm{~km} \mathrm{~s}^{-1}$ are shown in Fig. 2. We have modelled the resulting

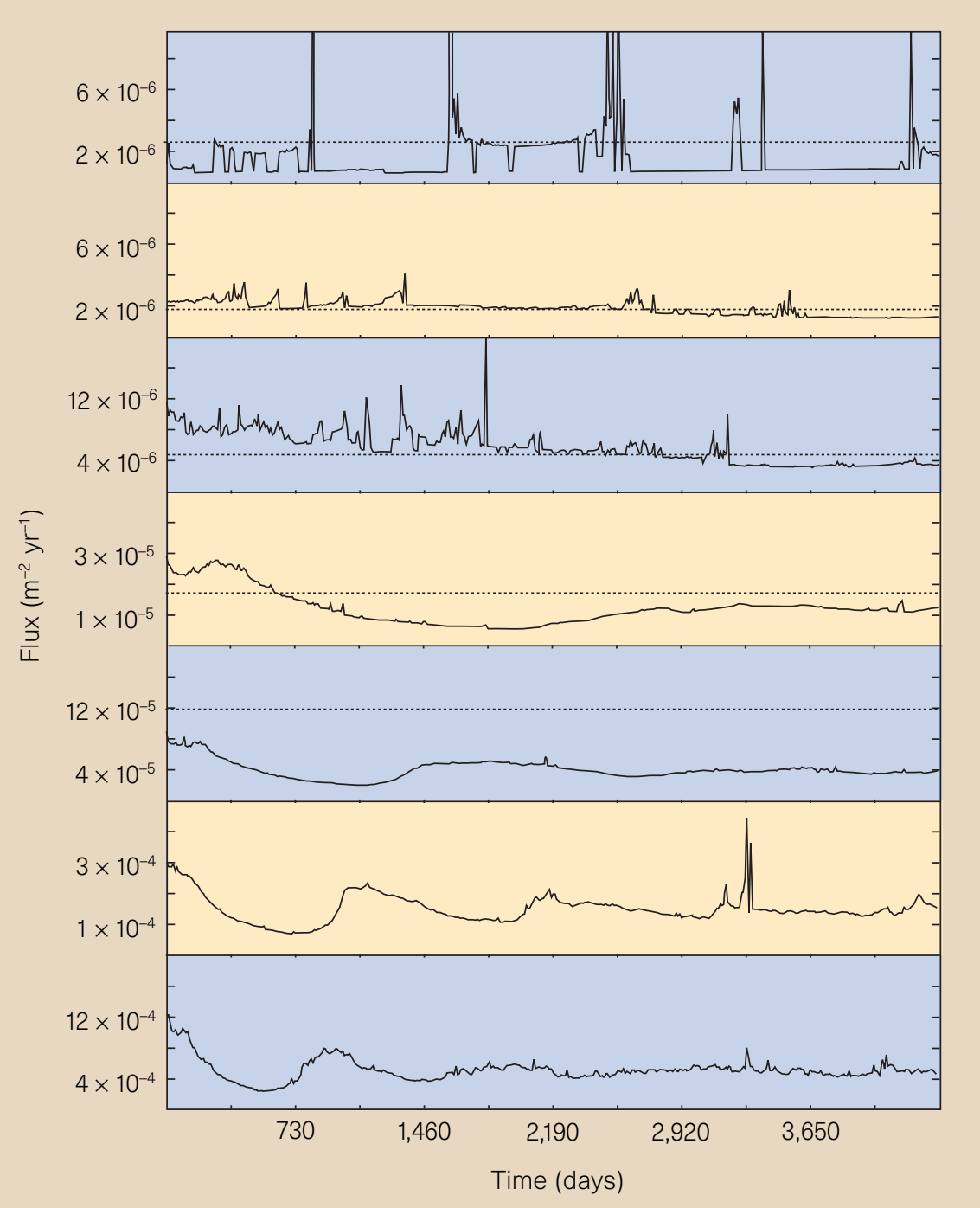

Figure 2 Flux of fragments over time produced by the simulated impact break-up of an Iridium satellite orbiting in plane number 6 of Fig. 1. Panels show, from top to bottom, the fluxes at decreasing kinetic energy, $E$ : $2 E>10^{9} \mathrm{~J} ; 10^{8}<2 E<10^{9} \mathrm{~J} ; 10^{7}<2 E<10^{8} \mathrm{~J} ; 10^{6}<2 E<10^{7} \mathrm{~J} ; 10^{5}<2 E<10^{6} \mathrm{~J} ; 10^{4}<2 E<10^{5}$ J; and $10^{3}<2 E<10^{4} \mathrm{~J}$. The target satellite is located in plane number 1 (Fig. 1). Horizontal dotted lines represent background flux in the same energy ranges, calculated from the overall space debris population, according to ref. 2 .

fragment swarm $(8,000$ bodies heavier than $1 \mathrm{~g})$ under Earth's oblateness and air drag perturbations, and computed as a function of time the cumulative probability of impact with another constellation satellite moving in the opposite direction.

For an impact energy between $10^{7}$ and $10^{8} \mathrm{~J}$, corresponding to such projectiles, the collision probability stays higher than the background level from the general orbiting population for several years. This means that, after the initial break-up, the probability that a second one will follow within five years is about $10 \%$. This may then trigger a chain-reaction effect with a characteristic timescale of about a century, much less than the current estimates with the general debris population (300 to $500 \mathrm{yr})^{10}$.

A. Rossi ${ }^{\star}$, G. B. Valsecchi†, P. Farinella ${ }^{*}$ CNUCE-CNR, Via S. Maria 36,

56126 Pisa, Italy

e-mail:a.rossi@cnuce.cnr.it
$† I A S-C N R$, Via Fosso del Cavaliere,

00133 Roma, Italy

$\ddagger$ Dipartimento di Astronomia,

Universita' di Trieste, 34131 Trieste, Italy

1. Rossi, A. \& Farinella, P. ESA J. 16, 339-348 (1992).

2. Pardini, C., Anselmo, L., Rossi, A., Cordelli, A. \& Farinella, P. Adv. Astronaut. Sci. 99, 1041-1058 (1998).

3. Kessler, D. J. et al. NASA Technical Memorandum 104825 (NASA, 1996).

4. Kessler, D. J. \& Cour-Palais, B. G. J. Geophys. Res. 83, 2637-2646 (1978).

5. Rossi, A., Cordelli, A., Farinella, P. \& Anselmo, L. J. Geophys. Res. 99, 23195-23210 (1994)

6. van der Ha, J. C. (ed.) Mission Design and Implementation of Satellite Constellations (Kluwer, Dordrecht, 1998).

7. McKnight, D. S., Maher, R. L. \& Nagl, L. Fragmentation Algorithms for Satellite Targets (FAST) Empirical Breakup Model, Version 2 (Kaman Sciences, 1992).

8. Walker, R., Crowther, R., Wilkinson, J., Stokes, P. H. \& Swinerd, G. G. in Mission Design and Implementation of Satellite Constellations (ed. van der Ha, J. C.) 317-326 (Kluwer, Dordrecht, 1998).

9. Rossi, A., Pardini, C., Anselmo, L., Cordelli, A. \& Farinella, P. in Mission Design and Implementation of Satellite Constellations (ed. van der Ha, J. C.) 327-335 (Kluwer, Dordrecht, 1998). 10. Cordelli, A., Farinella, P. \& Rossi, A. Planet. Space Sci. $\mathbf{4 6}$ 691-699 (1998). 